\title{
DEVELOPMENT OF FUEL CHARACTERIZATION TOOL BASED ON LIBRARY INTERPOLATION
}

\author{
Malec $\mathbf{J}^{1}$, Ambrožič $\mathbf{K}^{1}$ and Kromar $\mathbf{M}^{1}$ \\ ${ }^{1}$ Reactor Physics Department, \\ Jamova cesta 39, 1000 Ljubljana, Slovenia \\ jan.malec@ijs.si,klemen.ambrozic@ijs.si,marjan.kromar@ijs.si
}

\begin{abstract}
The feasibility of using Origen+ARP code for depletion and decay calculations for Krško NPP was tested by performing depletion and decay calculations using interpolated libraries and comparing the results to the ones calculated from non-interpolated libraries in order to evaluated the number of libraries needed in order to interpolate fuel properties with sufficient precision for any realistic burnup scenario. For Krško NPP fuel, using three interpolation libraries with different decay heat parameters was enough to bring the approximation error bellow $0.5 \%$ when comparing fuel decay heat through the decay interval.
\end{abstract}

KEYWORDS: depletion, decay, interpolation, SCALE

\section{INTRODUCTION}

Krško Nuclear Power Plant (NPP) is planning to build a dry storage facility for nuclear fuel that is currently being stored in a spent fuel pool. The motivation is to build a storage facility that does not require any active systems. We have recently completed a sensitivity analysis, where effects of different factors, such as burnup, fuel enrichment and fuel temperatures during burnup, on the fuel properties were estimated by depletion and decay simulations with the Monte Carlo neutron transport code Serpent2 [1] and different modules from the SCALE package [2]. In the SCALE main depletion was achieved with deterministic neutron transport sequence Triton, where the NEWT code is coupled to the Origen code. In addition stand-alone application of the Origen and Origami codes, which allows the calculation of depletion based on the pre-calculated one-group reaction coefficients library was analysed. Our department is currently developing an automatization process for the fast determination of the decay heat, activity, photon and neutron source term of the NPP Krško fuel assemblies. The tool will be based on the SCALE package and in-house developed core design package CORD-2 [3]. As a result a large number of fuel assemblies with unique composition will be processed quickly. The idea behind the tool is to calculate multiple reaction coefficients libraries with different fuel characteristics and use interpolation between libraries to calculate decay heat, activity, photon source term and neutron source terms for any NPP Krško assembly without re-doing neutron transport calculations. In this paper several validation results based on comparisons using Serpent 2 and Triton will be presented. 


\section{Library generation with SCALE}

Reaction coefficients libraries used for interpolation are generated by the Triton. Triton is a multipurpose control module for transport, depletion and sensitivity analysis. On output, it generates two types of libraries. "f33" files are Origen libraries containing the transition matrices with one-group reaction coefficients used for depletion and decay. "f71" libraries are Triton and Origen results file that stores all of the material inventories at each depletion/decay step. In the work described in this article, the fuel assemblies are generated using NEWT, a 2-D discrete ordinates transport code. Figure 1 is the geometry representation of the basic fuel assembly with no IFBA (Integral Fuel Burnable Absorber) rods. The white cirles represent the fuel rods and the dark orange circles represent guide thimbles filled with water.

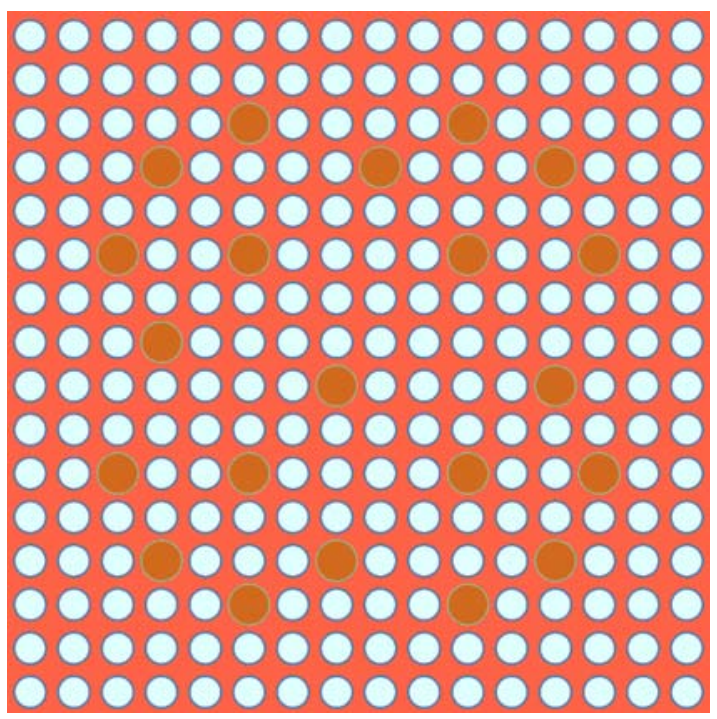

Figure 1: TRITON/NEWT Model of a NEK assembly with no IFBA fuel rods. The smaller, white circles represent fuel rods and the bigger orange tubes are zirconium guide thimbles filled with water.

\subsection{Comparison with Serpent 2}

Before the TRITON/NEWT generated libraries could be used for interpolation, a validation of NEWT results was needed to ensure that the results are accurate enough to be used for dry storage applications. The results are compared on identical cases computed with the Serpent2, a MonteCarlo code that is well validated for the PWR applications. Similar comparison have been previously conducted in the work reported in [4].

Figure 2 presents a comparison between results calculated from the TRITON/NEWT model of a NEK assembly with no IFBA rods shown on 1 and the Serpent 2 model of the same assembly. Bremsstrahlung radiation had to be disabled in the TRITON/NEWT model to match the results from the Serpent 2 simulation, since the Serpent 2 code is for now not capable to take it into account. It can be observed that the relative differences are smaller than $4 \%$, except for the Gamma emission rate at very low decay times. 


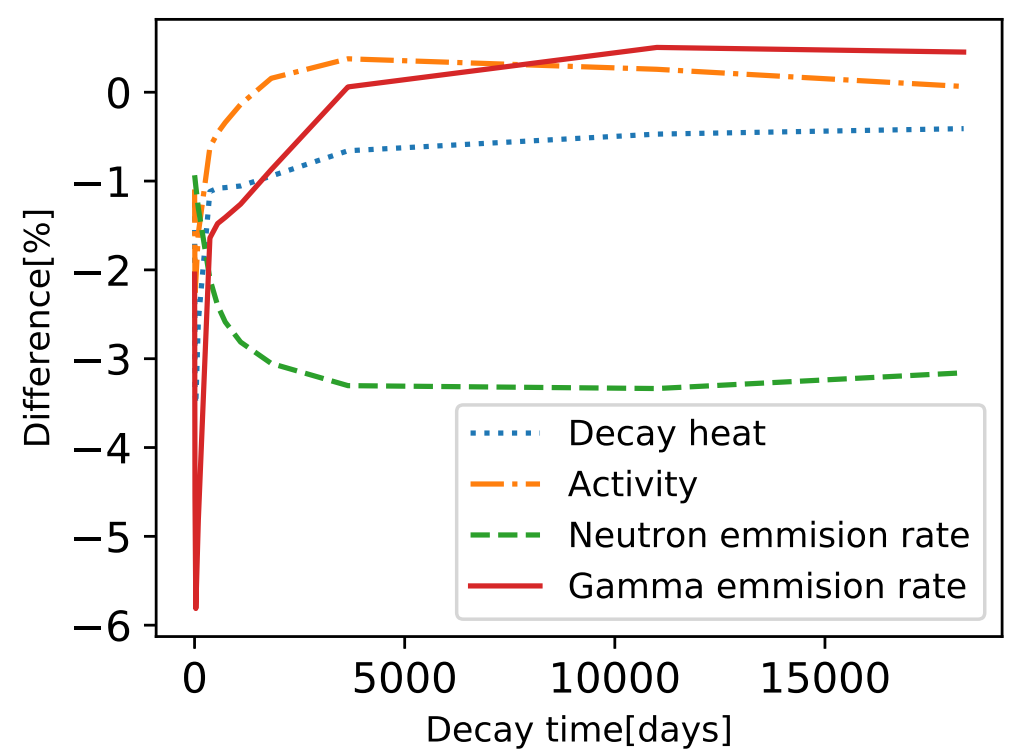

Figure 2: Comparison between TRITON/NEWT model of a NEK assembly with no IFBA fuel rods and Serpent 2 model

\section{Depletion and decay from Triton-generated libraries}

Before the "f33" libraries are used to interpolation, it have to be verified that the Triton results for decay heat, activity, gamma source and neutron source can be reproduced by using Origen and the "f33" libraries. In order to match the results, it is necessary to understand the Triton stepping algorithm. According to the SCALE manual [2], Triton uses a predictor corrector approach and performs processing of cross sections at the midpoint of each burnup interval. The Triton user has no control over the sub-step size or number of sub-steps.

During a typical calculation, Triton first performs particle transport and cross section processing at the initial zero time, followed by the depletion. It proceeds with a particle transport calculation and processing of cross sections at the midpoint of the interval. The cross sections computed at the midpoint of the interval are than used to perform depletion calculations from the beginning to the end of the depletion sub-interval.

Since the exact formula used in Triton is not outlined in the SCALE manual, it was not possible to use Origen to "replay" the Triton results exactly, until dr. William Wieselquist shared details of the algorithm on a Google Group forum [5]. The formula for number of sub-steps during each depletion interval is:

$$
\operatorname{nsub}=\max (4, \operatorname{int}(\operatorname{burn} / 40)+1)
$$

where nsub is the number of sub-steps and burn is the burnup increment during the user defined interval in the units of MWd/MTU. When time steps as defined in 1 are used in an Origen calculation, the results of the Triton and Origen are inside numerical precision and differ for less than $0.1 \%$. 


\section{Library interpolation}

In order to avoid costly neutron transport calculations, the Origen libraries containing transition matrices can be used for depletion and decay. SCALE provides a tool "ARP" intended to create Origen libraries by interpolating existing libraries using one of the following methods: Nearest value, Linear interpolation, Lagrangian interpolation, Standard cubic spline and Monothonic cubic spline.

The main purpose of the work presented here was to evaluate how many parameters need to be interpolated and how many libraries need to be pre-generated for interpolation per parameter to allow users to generate decay data for all fuel used in the Krško NPP without re-running neutron transport calculation.

In this work, Triton was used to generate perturbed libraries with varying enrichments, fuel temperatures, moderator temperatures and number of IFBA rods. The perturbed libraries were used to generate interpolated libraries using ARP, which were utilized in Origen depletion and decay calculations. The results were compared with the ones generated by using exact, non-interpolated libraries with the same parameters as in the case of the interpolated libraries.

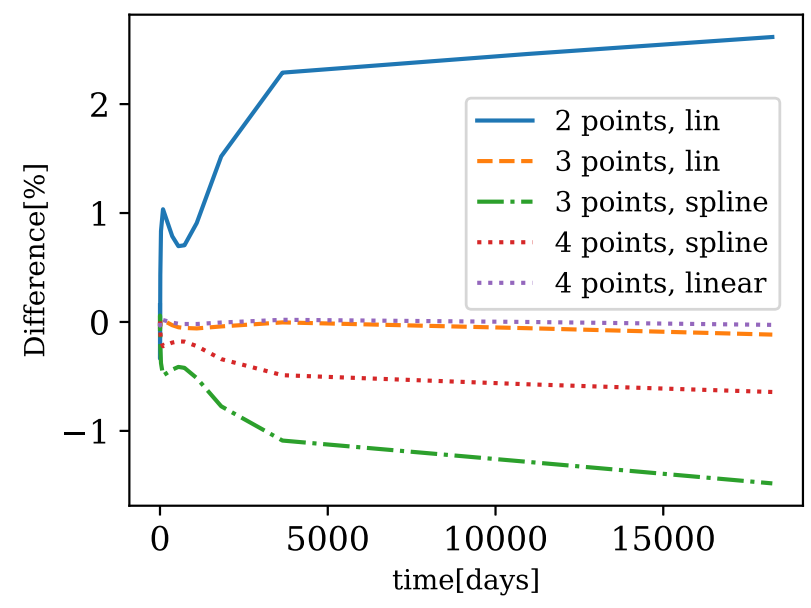

(a) 2 points: $2.1 \%$ and $4.95 \%$ enrichment interpolated to $3.525 \%$ enrichment, 3 points: $4.27 \%$ enr. added, 4 points: $2.73 \%$ enr. added

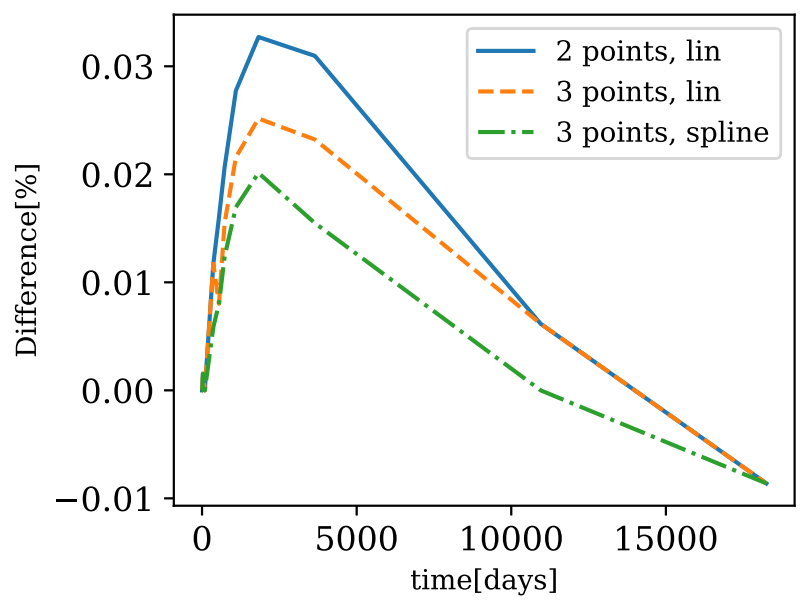

(b) 2 points: Fuel temperatures $800{ }^{\circ} \mathrm{C}$ and $1000{ }^{\circ} \mathrm{C}$ interpolated to temperature of $900{ }^{\circ} \mathrm{C}, 3$ points: 950 ${ }^{\circ} \mathrm{C}$ was added

Figure 3: Comparison of the error calculated by comparison of the decay heat during the fuel cooling.

Figure 3a shows the difference of the decay heat computed by using libraries interpolated from two, three and four points and compares results of linear interpolation with monotonic cubic spline [2]. All errors on plots in Figure 3 and Figure 4 are computed using the formula

$$
\text { Difference }=\left(1-\frac{\text { interpolated decay heat }}{\text { exact decay heat }}\right) .
$$

As expected, using a greater amount of libraries during interpolation results in a better approxima- 
tion. Surprisingly, when interpolating enrichment, linear interpolation yielded better results than cubic spline.

On Figure $3 b$ we can observe the results of different interpolation scenarios for fuel temperature. While the decay heat at the end of the cycle is not greatly sensitive to the fuel temperature during depletion, the temperature is a good independent parameter that can be used to test interpolation schemes. Again, using a greater number of interpolation points yields better results, but in this case Cubic spline interpolation yielded a better approximation than linear approximation.

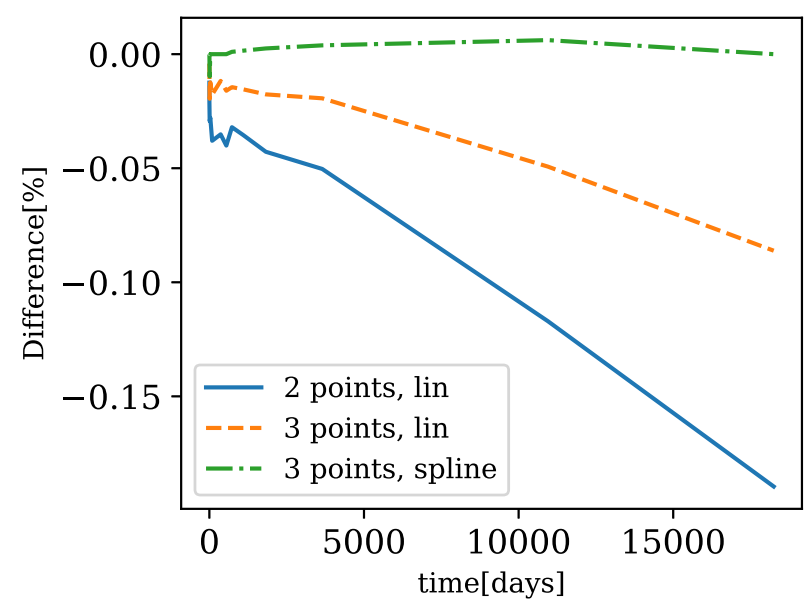

(a) 2 points: Temperatures 560 and 600 interpolated to 580,3 points: 590 was added

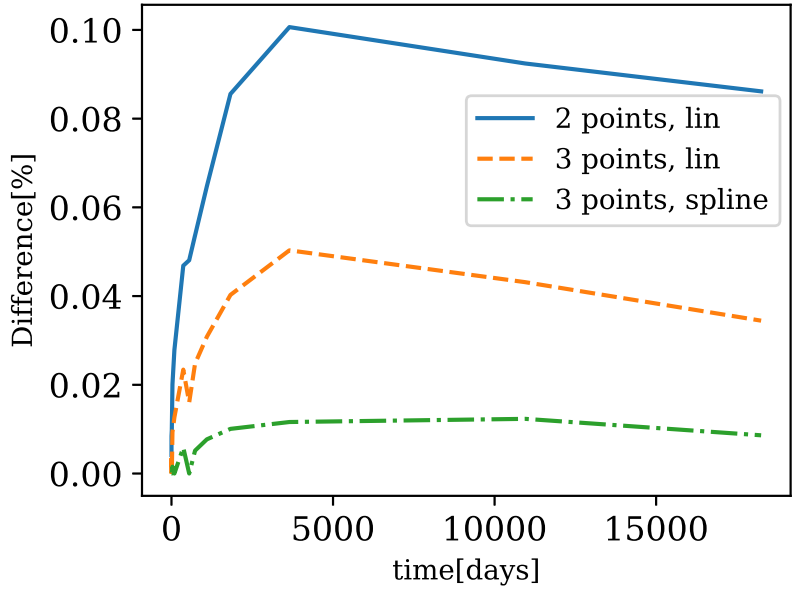

(b) Moderator densities calculated from temperatures on Figure 4a using formula from [6] and [3]

Figure 4: Comparison of the error calculated by comparing decay heat during fuel cooling.

Plots on Figure 4 depict a comparison of results gained after interpolating libraries based on moderator temperature on Figure $4 \mathrm{a}$ and moderator density on $4 \mathrm{~b}$. Both plots show a smaller error when using three interpolation points compared to two interpolation points and a smaller error with cubic spline interpolation compared to linear interpolation. What is more interesting is that better results are achieved when interpolating the libraries based on the moderator density compared to the interpolation based on the moderator temperature. While moderator density and temperature are inversely proportional, the relationship between the two quantities is not linear. The moderator temperature affects the core parameters mostly through changes in density causing the library interpolation through it more accurate.

In addition to the basic configuration with no IFBA rods, Krško NPP core design documentation for fuel cycle 31 defines five possible core arrangements with 20, 32, 80, 92 and 116 IFBA rods. The core configurations from previous cycles were not considered in this approximation. The decay heat, activity, gamma source and neutron source terms during decay of the irradiated fuel obviously also depend on the IFBA configuration. Since the parameters don't depend just on the number of IFBA rods in the fuel but also the arrangement of the rods in the assembly, the effect of the number of IFBA rods on physical properties of the irradiated fuel cannot be linear.

Figure 5 tells us how well can interpolated libraries of different IFBA configurations describe the exact configurations. By interpolating more than two points and using cubic spline interpolation, 


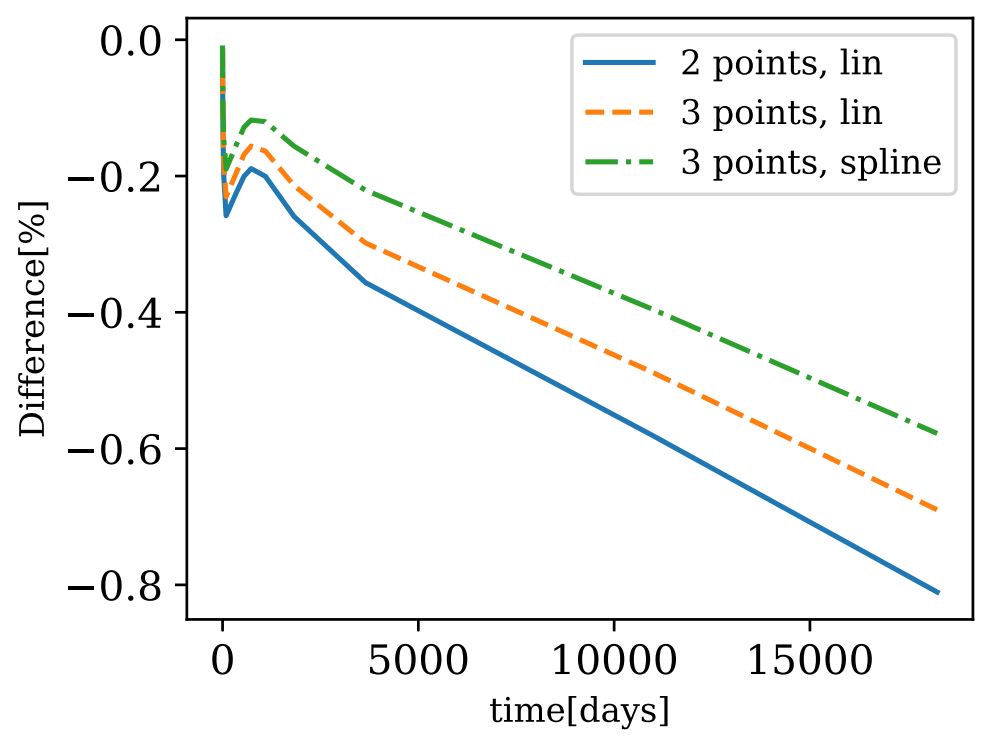

Figure 5: The interpolation of different IFBA configurations. During the two-library interpolation, libraries generated by simulating configurations with 20 and 116 IFBA rods were interpolated to a library with 90 core rods. In the 3 point interpolation, another library for a configuration with 32 IFBA rods is added.

an error less than $\approx 0.5 \%$ can be achieved. Interpolation of more than three points is not trivial, since arbitrary core configurations would have to be generated.

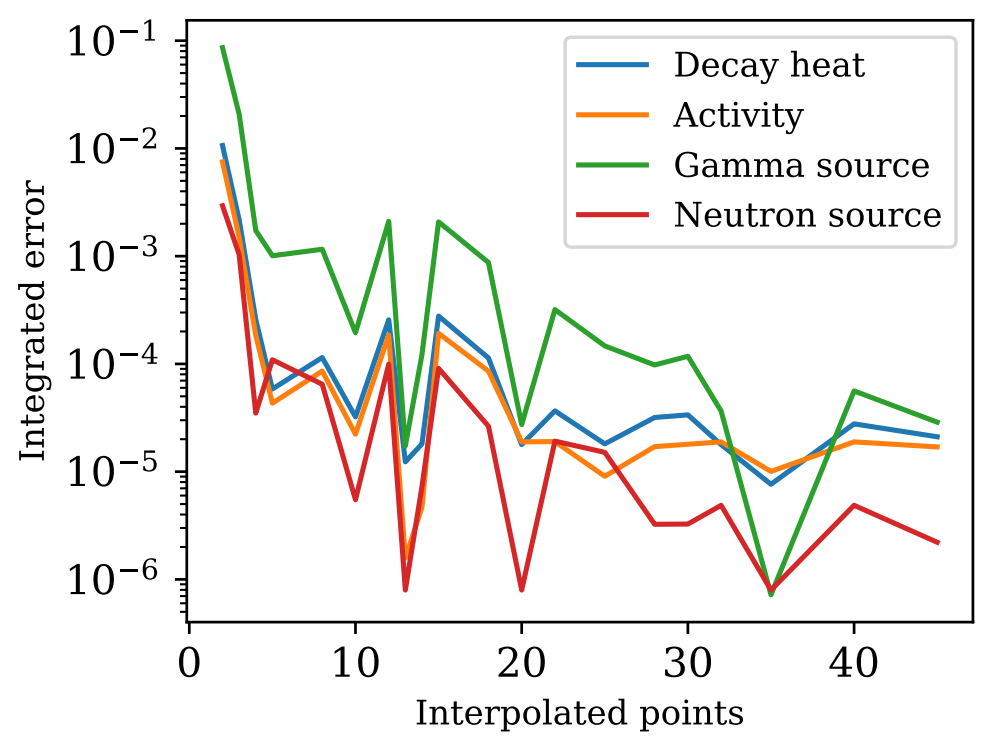

Figure 6: Mean absolute error during decay for different numbers of interpolated points. Each interpolation point is a library with reaction cross sections. All interpolations were computed using cubic spline method. 
The plot of the total error during fuel cooling as a function of the number of interpolated libraries is presented in Figure 6. The total error on the graph was calculated as the sum of absolute differences or $\mathrm{E}_{\text {total }}=\sum_{i}^{N} \mid$ difference $\mid / N$, where $\mathrm{E}_{\text {total }}$ is the quantity being plotted, $i$ goes from 1 to the number of decay steps and the difference is computed as in Eq. 2. The interpolated points were generated on equal intervals from the lower limit for enrichment of $2.1 \%$ to the higher limit of $4.95 \%$. The error is clearly falling as more points are used, but the trend is not strictly monotonic. Achieving errors lower than $10^{-6}$ might not be possible due to errors that are not directly connected to interpolation, but is also not necessary for many applications.

\subsection{Sources of errors}

The biggest and most obvious source of error comes from the interpolation itself. Figure 7 shows the deviation of the simulation result when interpolating two points in case of $7 \mathrm{a}$ with linear interpolation or three points in case of $7 \mathrm{~b}$ using cubic spline to another point inside the interpolation interval. In case of linear interpolation, any non-linear phenomena will result in excess errors. Similarly, the shape of too complex functions used in interpolation schemes could yield oscillations with computed functions very likely mismatching the physical phenomena. These errors can be minimized by interpolating at a denser grid of libraries, but the improvement is not always linear due to method limitations. In case of monotonic cubic spline interpolation, the points neighbouring the interpolated point will clearly have the greatest effect, all other points will result in a minor slope corrections.

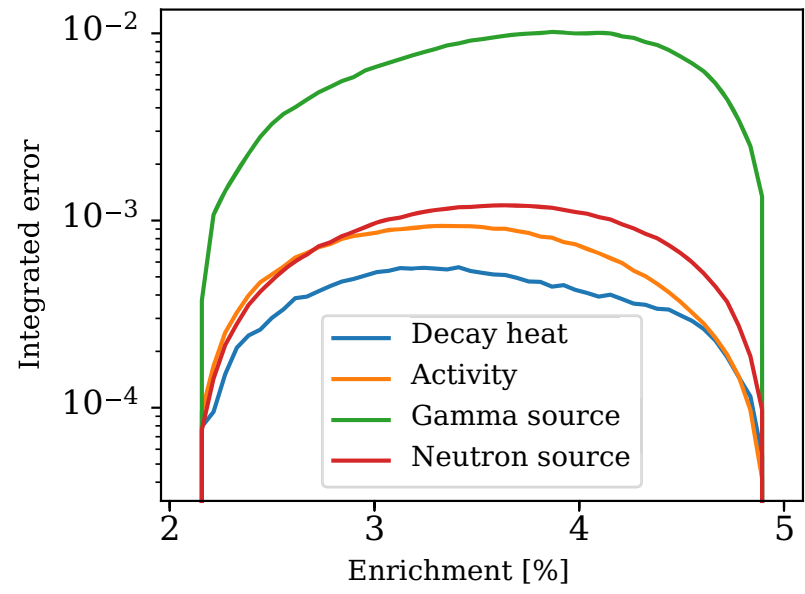

(a) Interpolation error with linear interpolation of two libraries with enrichments $2.1 \%$ and $4.95 \%$ to different values inside interval.

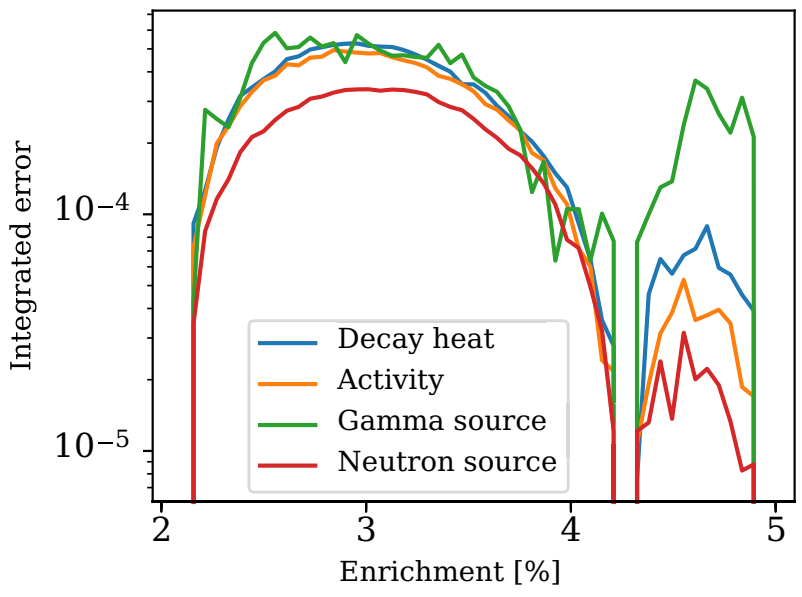

(b) Interpolation error with spline interpolation of three libraries with enrichments $2.1 \%, 4.27 \%$ and $4.95 \%$ to different values inside interval.

Figure 7: Error calculated by the comparison of the decay heat during fuel cooling.

Other sources of errors come from the way the tools are constructed. One of the limitations is that ARP requires a file named "arpdata.txt" that expects the exact fuel burnups for each burnup step to describe the libraries used for interpolation. Since the list of burnups is provided only once for all libraries used for interpolation, an assumption that all libraries have exactly equal burnup steps is being made. This is not always true, since the ratio of the burnup in the region containing the 
reactor fuel compared to the burnup of all materials in the system can differ slightly.

\section{CONCLUSIONS}

The main conclusion is that Origen+ARP can be effectively used for interpolation of the Origen one-group reaction coefficients libraries in practical applications where a large amount of neutron transport applications would be required otherwise. The number of libraries required to interpolate based on fuel enrichment, fuel temperature, moderator density and the number of IFBA rods was determined by interpolating the highest and lowest possible values for the NPP Krško fuel and adding equidistant points until a desired precision was achieved. In all the studied cases, three points were enough to achieve an error lower than $0.5 \%$. Triton generates libraries using mutilple sub-steps, so care must be taken when interpreting the libraries using other codes, such as ARP+Origen.

\section{REFERENCES}

[1] J. Leppänen, M. Pusa, T. Viitanen, V. Valtavirta, and T. Kaltiaisenaho. "The Serpent Monte Carlo code: Status, development and applications in 2013." Annals of Nuclear Energy, volume 82, pp. 142-150. URL http://www.sciencedirect.com/science/article/pii/ S0306454914004095.

[2] B. T. Rearden and M. A. Jessee. SCALE Code System. ORNL/TM-2005/39 Version 6.2.3. Oak Ridge National Laboratory, Oak Ridge, Tennessee, (2018). URL https://www.osti.gov/biblio/ 1426571-scale-code-system.

[3] M. Kromar and A. Trkov. "Nuclear design calculations of the NPP Krško core." Journal of energy technology, volume 2(4), pp. 41-50.

[4] M. Kromar and B. Kurinčič. "Determination of the NPP Krško spent fuel decay heat." AIP conference proceedings, pp. str. 050005-1. American Institute of Physics (2017).

[5] W. Wieselquist. "How can one verify equivalence between TRITON, ORIGEN, and ORIGAMI depletion results?,' SCALE Users Groups.” (Feb. 2019). URL https://groups. google.com/forum/\#!msg/scale-users-group/StjdvKo6MIQ/JtqwRfihAgAJ.

[6] E. Schmidt and U. Grigull. Properties of water and steam in SI-units:. Springer (1981). URL https://books.google.si/books?id=nOrkAAAAMAAJ. 